\title{
Value of Information Introduced by a Resource Model Updating Framework
}

\author{
Cansın Yüksel $^{1}$ (D) Corinna Minnecker ${ }^{2}$. \\ Masoud Soleymani Shishvan ${ }^{1}$ - Jörg Benndorf ${ }^{2}$. \\ Mike Buxton ${ }^{1}$
}

Received: 30 October 2017 / Accepted: 14 October 2018 / Published online: 8 November 2018 (C) The Author(s) 2018

\begin{abstract}
A primary concern of the mining industry is to meet production targets, which are required and defined by customers. Deviations from these targets, in terms of quality and quantity, highly affect the economical aspect. Recently, an efficient resource model updating framework concept has been proposed aiming for the improvement of raw material quality control and process efficiency in any type of mining operation. The concept integrates online sensor measurements, obtained during production, into the resource model. In this way, due to the spatial variability, quality attributes of the blocks that will be produced in the next days or weeks are being updated based on real-time measurements. The concept has been applied in a lignite field with the aim of identifying local impurities in a lignite seam and to improve the prediction of coal quality attributes in neighbouring blocks. This paper investigates the added value of using the resource model updating framework by using the value of information analysis. The expected benefit of additional information (integration of the online sensor measurements into the resource model) is compared to a case where there is no additional information integrated into the process. These benefits are evaluated based on the economic impact determined by applying the resource model updating framework in mine planning.
\end{abstract}

Keywords Resource model updating · Optimization - Simulation · Lignite $\cdot$ Real application · Mine planning

$\bowtie$ Cansın Yüksel

C.Yuksel@tudelft.nl

1 Resource Engineering Section, Department of Geoscience and Engineering, Delft University of Technology, Stevinweg 1, 2628 CN Delft, Netherlands

2 Institute for Mine Surveying and Geodesy, Faculty of Geosciences, Geoengineering and Mining, University of Technology Bergakademie Freiberg, Reiche Zeche, Fuchsmühlenweg 9 B, 09599 Freiberg, Germany 


\section{Introduction}

One of the main challenges in mining is spatially highly variable grades or quality attributes of the resource. Extreme values or impurities cannot be localized completely by exploration data and therefore cannot be captured and predicted in deposit models. Utilizing online sensor technique characterization in combination with rapid resource model updating, a faster reaction to unexpected deviations can be implemented during operations, leading to locally improved models and thus increased production efficiency. This concept was first proposed as a closed-loop framework and applied to a coal deposit by Benndorf et al. (2015). The developed framework is based on the ensemble Kalman filter (EnKF) and integrates online sensor data into the resource model as soon as data are obtained.

A first investigation (Benndorf 2015) has proven the approach to work well within a synthetic case study under a variation of several control parameters. Wambeke and Benndorf (2016) extended the framework for practical applications, including the handling of attributes and measurements showing a non-Gaussian distribution, dealing with localization and inbreeding issues, avoiding spurious correlations and increasing the computational efficiency. The third investigation (Yüksel et al. 2016a, b) implemented the framework in a full case study by adapting implementation details for coal quality attributes in a continuous mining environment. The framework's applicability for a full-scale lignite production environment was validated, and significant improvements were demonstrated. These results have been demonstrated for a case where one sensor has been placed on an excavator. This sensor observes the produced material from that excavator and the data produced by this sensor is being used for updating the neighbourhood blocks around the mined blocks. An extension was presented by Yüksel et al. $(2016 a, b)$ who presented a new application of the framework in a full-scale lignite production, where the initial resource model generation is semi-automated without the explicit need of geostatistically simulating prior models. This application allows for a fast and rather simple generation of prior models instead of generating a fully simulated deposit model using conditional simulation. Additional to that, the contribution provided a real updating application of local coal quality estimates in different production benches based on measurements of a blended material stream. To investigate the impact of the improvements achieved by the previously mentions applications, this contribution aims to quantify the economic impacts determined by applying the resource model updating framework in mine planning.

One of the commonly used tools for assessing the value of additional information added into a system is the value of information (VOI; Howard 1966; Raiffa 1968; Matheson 1990). In the last decades, VOI gained high popularity in many different fields. A few applications also appeared in the mining industry. Peck and Gray (1999) make no explicit reference to VOI, yet they discuss the potential benefits for decision makers by gathering information in the mining industry. Barnes (1986) applied VOI to incorporate geostatistical estimation into mine planning. More recently, Phillips et al. (2009) provided a case study where a VOI decision framework was applied to provide guidance for mine managers regarding the purchase of ore grade scanners. Eidsvik and Ellefmo (2013) conducted a VOI study in order to compare two grade 


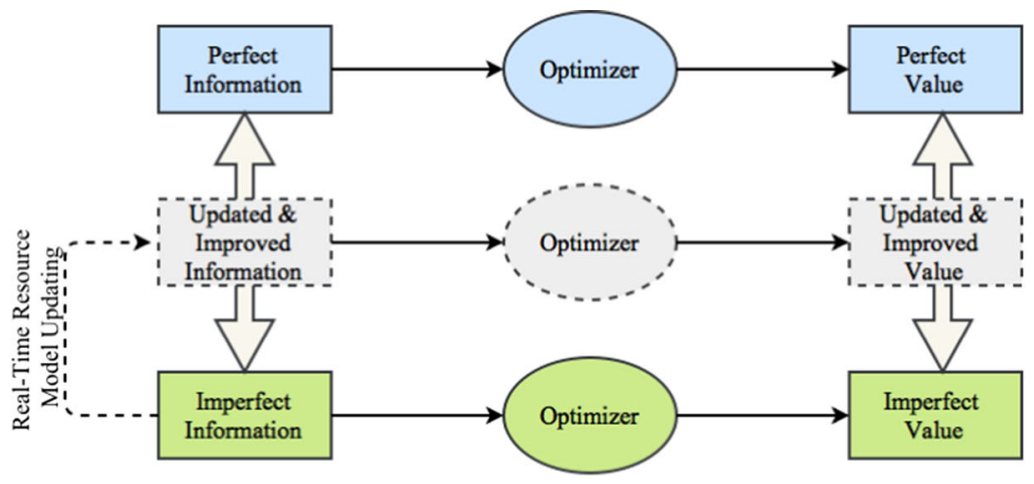

Current

Situation

Fig. 1 Aim of the resource model updating framework

analysing methods (differing in costs) in the collected data from exploration boreholes. Eidsvik et al. (2016) presented a unified framework for assessing the value of potential data-gathering schemes by integrating spatial modelling and decision analysis, with a focus on petroleum, mining and environmental geosciences. Contrary to the mining industry, the VOI approach has found more applications in a related field, the oil and gas industry. Bratvold et al. (2009) provided an extensive overview on oil and gas industry applications. Bhattacharjya et al. (2010) integrated the decision-analytic notion of VOI with spatial statistical models, similar to this paper's application work. Barros et al. (2016) proposed a new methodology to perform VOI analysis within a closed-loop reservoir management framework, which is a similar framework of resource model updating, except it is applied in reservoir engineering. Further applications in the oil and gas industry can be found (Grayson 1960; Newendorp 1975; Stibolt and Lehman 1993; Houck 2004; Steagall et al. 2005; Bickel et al. 2008).

The essence of the technique is to evaluate the benefits of collecting additional information before making a decision (Bratvold et al. 2009). When using the resource model updating framework, the decision-making process would change the short-term mining plan by using a mine optimizer. If the resource model always provides correct coal quality attributes it would deliver perfect information, otherwise it is known as imperfect information. The latter is usually the case in geoscience applications, since the reality is unknown. The resource model updating framework aims to carry forward the current situation from imperfect information to an "improved" imperfect Information state, where the current situation lies somewhere between the perfect information and previous imperfect information (Fig. 1). The previous imperfect information state gets closer to the perfect information state with each iteration step of the updating process.

The expected benefit of additional information (integration of the online sensor measurements into the resource model) is compared to a case where there is no additional information integrated into the process. These benefits are evaluated based on the economic impact, for example, the monetary value such as cost per shift of mining operation, determined by applying the resource model updating framework in the mine planning process. This contribution addresses the following question: What is 
the value of integrating real-time production measurements into the resource model and executing an optimized mine plan, considering economic aspects?

The remainder of this article is structured as follows: First, the resource model updating framework is briefly reviewed. Next, the VOI concept is introduced. Then, the principles behind the stochastic-based mine process optimizers for short-term and weekly job scheduling mine planning are presented. This is followed by a case study performed in a full-scale lignite production. Results are discussed and summarized. The article concludes with a summary of the research contributions and directions for future research.

\section{Updating Attributes in a Resource Model Based on Online Sensor Data}

For rapid updating of the resource model, sequentially observed data must be integrated with prediction models in an efficient way. For this study, this is done by using sequential data assimilation methods, namely the EnKF-based methods. A formal description of the real-time updating algorithm used herein is provided in Yüksel et al. (2016a, b). Figure 2 provides a general overview of the operations performed to apply the updating algorithm for improving the coal quality control using online data. The concept initially starts with resource modelling by using a geostatistical simulation technique, namely the sequential Gaussian simulation (SGS). This is the first required data set consisting of ensemble members to be updated. The second data set consists of a collection of actual and predicted sensor measurements. The actual online sensor measurement values are collected during the lignite production and the predicted measurements are obtained by applying the production sequence as a forward predictor to the prior resource model realizations. Once both input data are provided, the updated posterior resource model can be obtained. This process will continue as long as the online sensor measurement data are received.

\section{Value of Information}

In the context of this contribution, the VOI concept is used to understand what is gained by integrating the online sensor measurement data into the resource model when using the updating framework. In general, VOI is calculated as

$$
\mathrm{VOI}=\left[\begin{array}{c}
\text { Expected value with } \\
\text { additional information }
\end{array}\right]-\left[\begin{array}{l}
\text { Expected value without } \\
\text { additional information }
\end{array}\right],
$$

defined by Bratvold et al. (2009). In the coal-based case study presented later, the concept analyses the value of the resource model updating framework's ability to improve the prediction of the ash percentage (ash \%). For this, the expected value of the posterior model ( $\left.V_{\text {posterior }}\right)$ is compared to the prior model's expected value $\left(V_{\text {prior }}\right)$ and it is

$$
\mathrm{VOI}=V_{\text {posterior }}-V_{\text {prior }}
$$






Fig. 2 Configuration of the real-time resource model updating concept, modified from Wambeke and Benndorf (2015)

Translated into economic terms, the VOI [Eq. (3)] of the resource model updating framework can be expressed by

$$
\mathrm{VOI}_{\text {economical }}=\left|C_{\text {posterior }}-C_{\text {prior }}\right|
$$

where $\left|C_{\text {posterior }}-C_{\text {prior }}\right|$ denotes the absolute value. The performed research focuses on the costs of deviating from the target quality (ash \%) during short-term production. The calculation of these costs can be done as follows

$$
C_{\text {prior }}=D_{\text {prior }} * d_{\text {prior }} * t_{\text {prior }}
$$

where $C_{\text {prior }}(€)$ is the costs of deviating from the target production quality, when executing the mine plan on the prior model. The unit costs for deviating per ton of coal is $D_{\text {prior }}(€ /$ ash $\% \times t)$. The amount of deviation per coal quality value is $d_{\text {prior }}$ (ash \%), and, finally, the amount of the deviated coal is $t_{\text {prior }}$ (ton). Similar applies to the posterior model. The previously defined parameters are: $C_{\text {posterior }}, D_{\text {posterior }}$, $d_{\text {posterior }}$, and $t_{\text {posterior }}$.

The VOI concept considers the value of perfect and imperfect information (VOPI). Perfect information refers to perfectly reliable information; thus, it contains no uncertainties. Perfect information rarely exists, but it provides a best-case scenario for the VOI, and it defines an upper limit on the value of additional information (Phillips et al. 2009). Since the study presented here presents a real case, the reality remains unknown. Thus, there can be no VOPI defined. As a benchmark, a resource model is used that integrates all available sensor information available to the end of the study. As indicated in Fig. 1, the performed experiments will compare the calculated VOIs 
Table 1 Characteristics of the short-term and weekly job scheduling mine planning optimization models

\begin{tabular}{|c|c|c|}
\hline Model & Short-term mine planning & Weekly job scheduling \\
\hline Task & Monthly mine plan & Weekly/daily mine plan \\
\hline Approach & $\begin{array}{l}\text { Mixed integer programming to } \\
\text { reproduce the mining operation } \\
\text { and determining the best } \\
\text { configuration for a set of } \\
\text { decision variables }\end{array}$ & $\begin{array}{l}\text { Discrete event simulation to } \\
\text { simulate the mining operation } \\
\text { in combination with a hybrid } \\
\text { genetic and simulated } \\
\text { annealing search algorithm } \\
\text { determining the best } \\
\text { configuration for a set of } \\
\text { decision variables }\end{array}$ \\
\hline Objectives & $\begin{array}{l}\text { Meeting weekly coal tonnages } \\
\text { and grade targets }\end{array}$ & $\begin{array}{l}\text { Meeting daily coal tonnages and } \\
\text { grade targets }\end{array}$ \\
\hline \multirow[t]{4}{*}{ Constraints } & $\begin{array}{l}\text { Planned equipment's } \\
\text { maintenance schedule } \\
\text { (equipment availability) }\end{array}$ & $\begin{array}{l}\text { Planned equipment's } \\
\text { maintenance schedule } \\
\text { (equipment availability) }\end{array}$ \\
\hline & $\begin{array}{l}\text { Equipment capacities } \\
\text { (production rates) }\end{array}$ & $\begin{array}{l}\text { Equipment capacities } \\
\text { (production rates) }\end{array}$ \\
\hline & $\begin{array}{l}\text { Precedence relationships within } \\
\text { a bench }\end{array}$ & $\begin{array}{l}\text { Extraction sequence in different } \\
\text { benches }\end{array}$ \\
\hline & $\begin{array}{l}\text { Required equipment downtimes } \\
\text { while belt shifting occurs }\end{array}$ & \\
\hline \multirow[t]{3}{*}{ Decisions } & $\begin{array}{l}\text { Extraction sequence in different } \\
\text { benches }\end{array}$ & Excavator task scheduling \\
\hline & Belt shift scheduling & Blending of coal product \\
\hline & Blending of coal product & \\
\hline Time steps & Weeks & Shifts \\
\hline
\end{tabular}

of the imperfect value and updated \& improved value. These values will be calculated after applying the mine optimizers, described in the next section, on the prior and posterior (updated) models.

\section{Mine Planning Optimization}

In order to investigate the expected benefit of the updating framework, the framework is applied in the short-term mine planning context. To cover the short-term mine planning horizons, two different simulation-based optimizers were considered, which have been implemented within the context of the European Project RTRO-Coal (Benndorf et al. 2015; Table 1). Both models are used as a transfer function needed for the VOI concept as introduced in Fig. 1. The weekly job scheduling optimizer considers shiftbased scheduling (a shift being $8 \mathrm{~h}$ long), and it shows the benefits of the updating framework - to quickly integrate newly gained information into production control. In contrast, the short-term optimizer for extraction sequencing is used to demonstrate the benefits on a broader, less localized, scale and a longer time frame of months. 
Table 2 Illustration of how the binary decision variables determine the extraction sequence

\begin{tabular}{|c|c|c|c|c|}
\hline \multirow[t]{2}{*}{ Block } & \multicolumn{4}{|c|}{ Extraction period } \\
\hline & 1 & 2 & 3 & 4 \\
\hline 1 & 0 & 0 & 1 & 0 \\
\hline 2 & 0 & 0 & 0 & 0 \\
\hline
\end{tabular}

While block 1 will be mined in extraction period 3, block 2 is not scheduled for mining during extraction periods 1 through 4

\subsection{Short-Term Mine Planning}

Methods of mathematical optimization have been successfully applied in mining to find optimal solutions to various mine planning problems addressing issues on risk reduction and meeting production targets (Goodfellow and Dimitrakopoulos 2017; Liu and Reynolds 2017; Aliyev and Durlofsky 2017; Benndorf and Dimitrakopoulos 2013; Dimitrakopoulos 2011; Dimitrakopoulos and Ramazan 2004; Ramazan and Dimitrakopoulos 2007, 2013).

Like these optimization models, a short-term optimization model

$$
\min \left\{c^{T} x \mid A x \leq b, x \geq 0, x_{i} \in\{Z, R\}\right\}
$$

was formulated based on the mixed integer programming approach for the continuous lignite mining operation at hand. The mining process is represented by a system of linear (in-)equalities $A x \leq b$ and a set/vector of decision variables $x$ defining a mining plan. These linear inequalities are also referred to as constraints, and consider limits imposed by the operation such as the amount of extractable material per time period due to the deployed equipment. Each decision variable $x_{i}$ can either be binary (to determine the extraction sequence, Table 2) or continuous (to determine coal blending and when a belt shift should occur).

Which mining plan (value combination of the decision variables $x$ ) is the best is determined by the objective function $c^{T} x$. For short-term mine planning, deviations from production targets shall be minimized when excavating material from the mine according to the plan. Production targets are in terms of coal tonnage and coal quality (e.g., ash content) to ensure the reliable and continuous delivery of in-spec coal to the customers. Therefore, penalty functions for not meeting production targets have been defined (Fig. 3). Figure 3a shows a steeper slope for underproduction in comparison to overproduction since in this instance there is not enough lignite provided to the customer for example, endangering the correct operation of a power plant. In contrast, overproduction is not viewed as critical as underproduction because the considered mining operation has a lignite bunker at its disposal to temporarily store a limited amount of lignite to compensate for such things or standstills due to holidays, equipment failure, etc. 


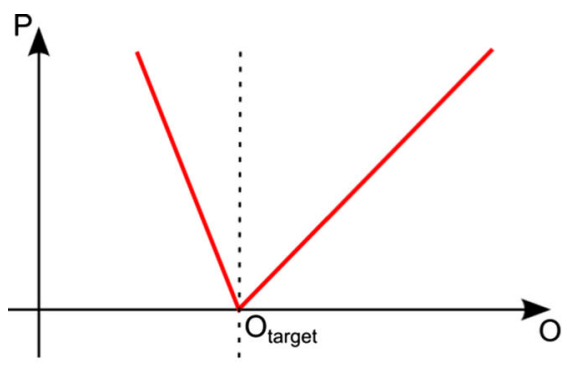

(a)

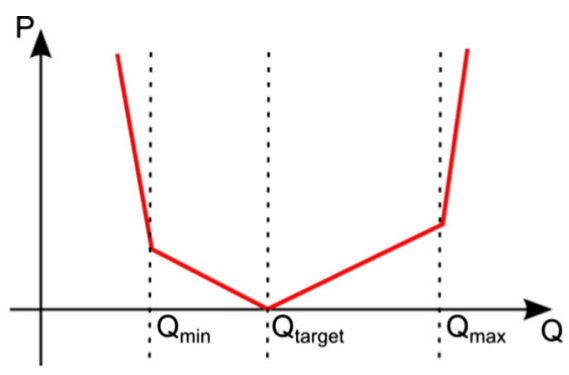

(b)

Fig. 3 Penalty functions for not meeting production targets a coal tonnage and $\mathbf{b}$ coal quality

The penalties for deviations in coal tonnage from the production target $O_{\text {target }}$ can be calculated in a similar way as the coal quality penalties, which are explained in detail in the next paragraphs.

For the coal quality production target (ash \%), the penalty function is a linear piecewise function consisting of four segments with slopes $\mathrm{sl}_{1}, \mathrm{sl}_{2}, \mathrm{sl}_{3}$, and $\mathrm{sl}_{4}$ (€/ash $\% \times t)$. The lower and upper limit of a coal quality parameter $Q_{\min }$ and $Q_{\max }$ are considered, which represent the maximum bandwidth for an efficient operation of the downstream process, such as power plants. Exceeding these thresholds will be penalized stronger than just slightly deviating from the target value $Q_{\text {target }}$. The penalty is calculated from

$$
P= \begin{cases}\operatorname{sl}_{1}\left(Q-Q_{\min }\right)+\mathrm{sl}_{2}\left(Q_{\text {min }}-Q_{\text {target }}\right) & Q \leq Q_{\text {min }} \\ \operatorname{sl}_{2} *\left(Q-Q_{\text {target }}\right) & Q_{\text {min }}<Q \leq Q_{\text {target }} \\ \operatorname{sl}_{3} *\left(Q-Q_{\text {target }}\right) & Q_{\text {target }}<Q \leq Q_{\max } \\ \operatorname{sl}_{4}\left(Q-Q_{\max }\right)+\operatorname{sl}_{3}\left(Q_{\max }-Q_{\text {target }}\right) & Q_{\max }<Q\end{cases}
$$

where $Q$ is the simulated quality.

To incorporate the resource model uncertainties, a neutral risk approach has been selected by calculating the penalty value for each simulated quality value of the resource model and then determining the mean of these penalty values $\bar{P}$. Then, the calculation of the costs for deviating from the target production quality value defined in Eq. (4) can be converted to

$$
C_{\text {prior }}=\bar{P}_{\text {prior }} * t_{\text {prior }}
$$

for the prior resource model. For the posterior model, this calculation is done in a similar manner.

\subsection{Weekly Job Scheduling}

Due to the complexity of interacting constraints in production scheduling, for weekly job scheduling, a flexible approach based on discrete event simulation and a hybrid genetic and simulated annealing search algorithm has been implemented. A simulation 


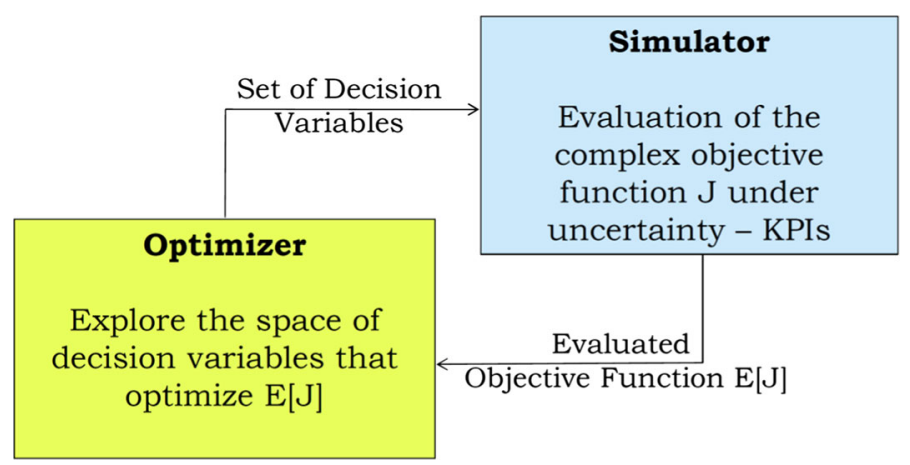

Fig. 4 Interaction between an optimizer and a simulator (Benndorf et al. 2015)

\begin{tabular}{|c|c|c|c|c|c|c|c|c|c|c|c|c|c|c|c|c|c|c|c|c|c|}
\hline \multicolumn{3}{|c|}{ Mo } & \multicolumn{3}{|c|}{ Tue } & \multicolumn{3}{|c|}{ We } & \multicolumn{3}{|c|}{ Thu } & \multicolumn{3}{|c|}{ Fri } & \multicolumn{3}{|c|}{ Sat } & \multicolumn{3}{|c|}{ Sun } & \multirow[b]{2}{*}{ Excavator 1} \\
\hline$x$ & $x$ & $x$ & & & $x$ & $\mathrm{x}$ & $x$ & $x$ & $x$ & $x$ & $x$ & $x$ & $x$ & $x$ & $x$ & $x$ & $x$ & & & & \\
\hline \multirow[t]{2}{*}{$x$} & $x$ & $x$ & $x$ & $x$ & $x$ & $\mathrm{x}$ & $x$ & $x$ & & & $x$ & $x$ & $x$ & $x$ & & & & & & & Excavator 2 \\
\hline & $x$ & $x$ & & $x$ & $x$ & & $x$ & $x$ & & $x$ & $x$ & & $x$ & $x$ & & $x$ & $x$ & & $x$ & $x$ & Excavator 3 \\
\hline \multirow[t]{3}{*}{$x$} & $x$ & $x$ & $x$ & $x$ & $x$ & $\mathrm{x}$ & $x$ & $x$ & $x$ & $x$ & $x$ & $x$ & $x$ & $x$ & $x$ & $x$ & $x$ & $x$ & $x$ & $x$ & Excavator 4 \\
\hline & $x$ & & & $x$ & & & $x$ & & & $x$ & & & $x$ & & & $x$ & & & $\mathrm{x}$ & & Excavator 5 \\
\hline & & & & & & & & & & & & $x$ & $x$ & $x$ & $x$ & $x$ & $x$ & $x$ & $x$ & $x$ & Excavator 6 \\
\hline$x$ & $\mathrm{x}$ & $\mathrm{x}$ & $x$ & $x$ & $\mathrm{x}$ & $x$ & $x$ & $\mathrm{x}$ & $\mathrm{x}$ & $x$ & $x$ & $x$ & $x$ & $x$ & $x$ & $x$ & $x$ & $\mathrm{x}$ & $\mathrm{x}$ & $x$ & Excavator 7 \\
\hline
\end{tabular}

Fig. 5 Visual representation of a series of task schedules, with seven simulation days and three shifts for each day. A cross means the excavator is scheduled to work (Mollema 2015)

model was developed to represent the complex continuous mining operation. This simulator serves as the objective function to evaluate different mining plans (value combinations of the decision variables $x$ ). To find the best mining plan, the simulator interacts with an optimizer. The optimizer explores the space of the decision variables and suggests a mining plan that is evaluated by the simulator. Based on the evaluation results, the optimizer choses which mining plan to evaluate next (Fig. 4).

For the weekly job scheduling process, the inputs/constraints to the simulator include, but are not limited to, the resource model, a given extraction sequence (mining plan), and the equipment's production rates and scheduled maintenance requirements. The decision variables of the optimization process are the task schedules of the excavators. The schedule for each excavator is a list with three shifts per day of the simulated period. For each shift, the excavator is either scheduled to work or is not active. The input for a single simulation is thus a two-dimensional array with six rows and $3 \times n$ columns, where $n$ is the number of simulated days. An example of a schedule is shown in Fig. 5 (Mollema 2015).

The stochastic mine optimizer also works with a penalty function to calculate the fitness of a solution. The total penalty value of a solution is the sum of both the quality and tonnage penalty value for all days of the simulation, similarly to the short-term optimization model. The structure of both penalty functions is similar to 


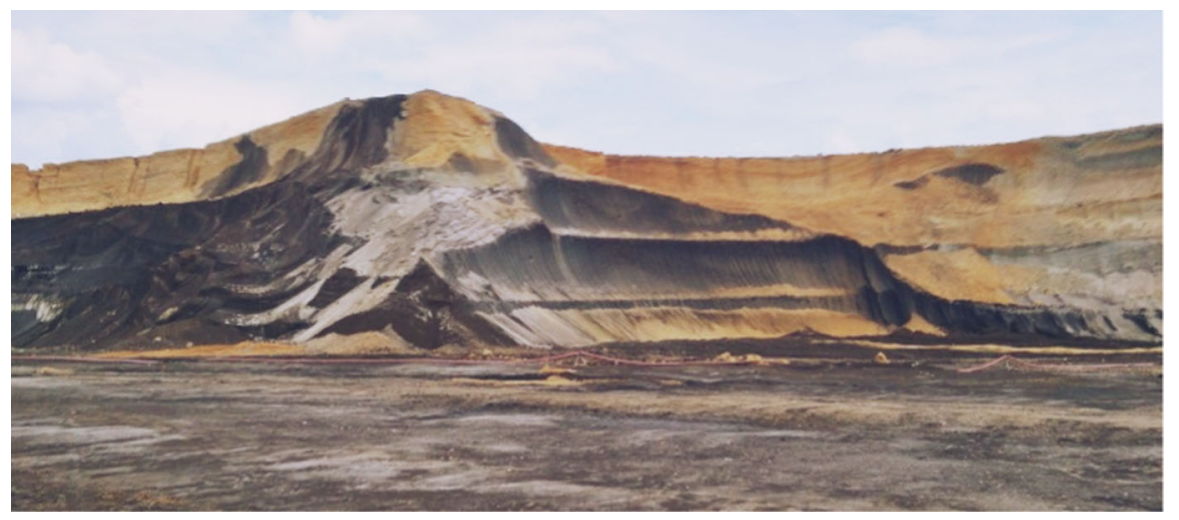

Fig. 6 Complicated geology in the lignite mine Profen (Germany)

the one depicted in Fig. 3b, except that the slopes for the inner segments are zero; reducing the number of segments, the piecewise linear function consists of from four to three. By minimizing the penalties, the optimizer tries to find a best task schedule. Thus, the resource model uncertainties are translated into penalty calculations based on an optimized mining schedule. For detailed information on the used mine process optimizer, the readers are referred to Mollema (2015). For calculating the penalty and the costs of deviating from the target production quality value, Eqs. (6) and (7) can be used.

\section{Case Study}

\subsection{Case Description}

The case study is performed on a lignite mining operation in Germany, where the geology of the field is complex, including multiple split seams with strongly varying seam geometry and coal quality distribution (Fig. 6). In this case study, the challenge originates from the complicated geology that leads to geological uncertainty associated with the detailed knowledge of the coal deposit. This uncertainty causes deviations from expected process performance and affects the sustainable supply of lignite to the customers. The knowledge of the coal deposit is improved, and the process performance is increased by applying the resource model updating framework described in previous works like Yüksel et al. (2016a, b). Now, the aim is to quantify the added value of the mentioned improvements in knowledge.

For the case study, the target area was defined as an already mined out area of $25 \mathrm{~km}^{2}$, where there are about 3000 drill holes. Mining operations are executed by six excavators, each working on a different bench. The produced materials are transported by conveyor belts. All conveyor belts merge at a central conveyor belt leading to the coal stock and blending yard, which is further connected to a train load. A radiometric sensor measurement (RGI) system is installed on the central conveyor belt just before the coal stock and blending yard (Fig. 7). This system allows an online determination of the ash content of the blended mass flow directly on the conveyor belt, without 

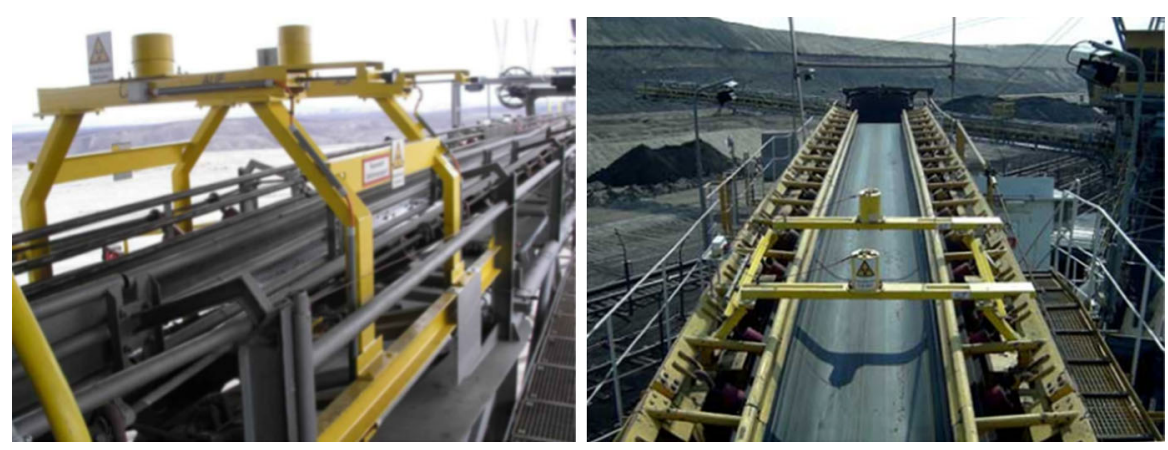

Fig. 7 Radiometric sensor measurement device

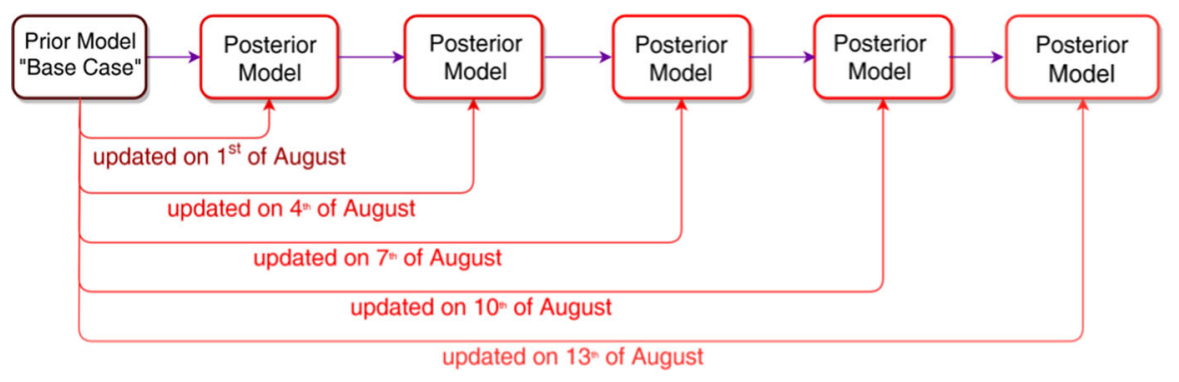

Fig. 8 Resource models that are used in the experiments

requiring any sampling or sample processing. This case study assumes the RGI values to be correctly calibrated and representative. The prior coal quality model has been generated by 25 realizations using geostatistical simulations (SGS) on a $25 \times 25 \times$ 1-m grid (SGS performed on each grid point) and afterwards relocked to a block size of about $50 \times 50 \mathrm{~m}$.

\subsection{Applying the Model Updating Framework}

The experiments performed in this case study calculate the expected values for different resource model-based experiments (Fig. 8). The base case resource model, namely the prior model, is without any additional information. The other resource models (posterior models) incorporate additional sensor information. They are created by applying the resource model updating algorithm up to different points in time. In total, there are five different posterior models representing different levels of information. This updating process is performed every $2 \mathrm{~h}$ using the actual mining sequence and the available RGI data for this time span. All the updating parameters are kept constant in order to compare the differences caused by feeding different prior models as an input.

The posterior model, which resulted from updating the prior model over the 19 July-13 August period, is assumed as the most precise model since this is the most up-to-date resource model created with the highest amount of exploration data, and therefore, it serves as a benchmark. 


\subsection{Weekly Job Scheduling Mine Planning}

The defined daily coal production is 12,000 tons and no penalties are applied for values between 9000 and 15,000 tons, a deviation of 3000 tons. The target production quality is defined as $9 \%$ ash and the penalty is only applied for the realizations above 10.5 ash \% and below 7.5 ash \%. The costs of deviating from the targets (the penalties) in this study are calculated by one unit per ton of coal. Hence, these penalties can be interpreted as percentage of deviation from the targets. A penalty of $0.1 € /$ ash $\%$ is applied per ton of coal. The mine optimizer is applied to all the resource models, defined in Sect. 5.2, for the following 5 days after their updating periods. For the base case and the benchmark case, the mine optimizer is applied for 5 days after each updating period. These dates will be 2-6 August, 5-9 August, 8-12 August, and 11-16 August. For these dates, there are different best schedules optimized based on the prior model, the updated model and the real model. Thus, in total, there are 12 different best schedules for different time spans.

Next, the best schedules obtained are applied to the benchmark model (Fig. 9). This is done to see the improvements during the mining operations when using the prior model, the most current model and the benchmark model. As mentioned in the previous section, the case study presented here is a real case study, and thus, there can be no VOPI defined for this case study. For this reason, the most precise model that incorporates most information is assumed to approximate reality. In this way, an approximation of VOPI can be calculated between the benchmark and the prior model, whereas the VOI is calculated between the posterior model and the prior model. A comparison of this would answer the following questions: "What would be the result of the mining activities if we didn't have additional information?", "How did the additional information affect the mining activities?" and, finally, "What would happen if we knew the reality and performed the mining activities based on that?" Of course, the latter one is only for comparison and, in reality, we can never have this information beforehand.

Once the optimized schedules are applied to the benchmark model, the expected costs of deviating from the target quality (ash \%) values will be calculated as explained in Sect. 4.1. The expected values are then compared to each other, and the VOI is calculated. This comparison and the results are provided next. Figure 10 presents the calculated deviation costs (penalties). This graph calculates the deviations per day for exceeding the upper target values of the ash content. Deviations from lower targets are of less interest. Figure 11 presents the calculated VOI for each case. The VOPI is represented with squared lines and the average of those VOPI is represented with a red line. The calculated VOI is represented with a pointed line and the trend line fitted on these points is represented with a dark green line.

In Fig. 10, the darkest column represents the calculated penalties for the optimized schedule based on the prior model. The lightest column represents the calculated penalties for the optimized schedule optimized based on the posterior model, which is updated until the mentioned time period. The medium darkest column represents the calculated penalties for the schedule using the benchmark model.

When evaluating the VOI for the next 5 days of mining after updating, a significant penalty reduction can be observed for the posterior model. This leads an increasing 


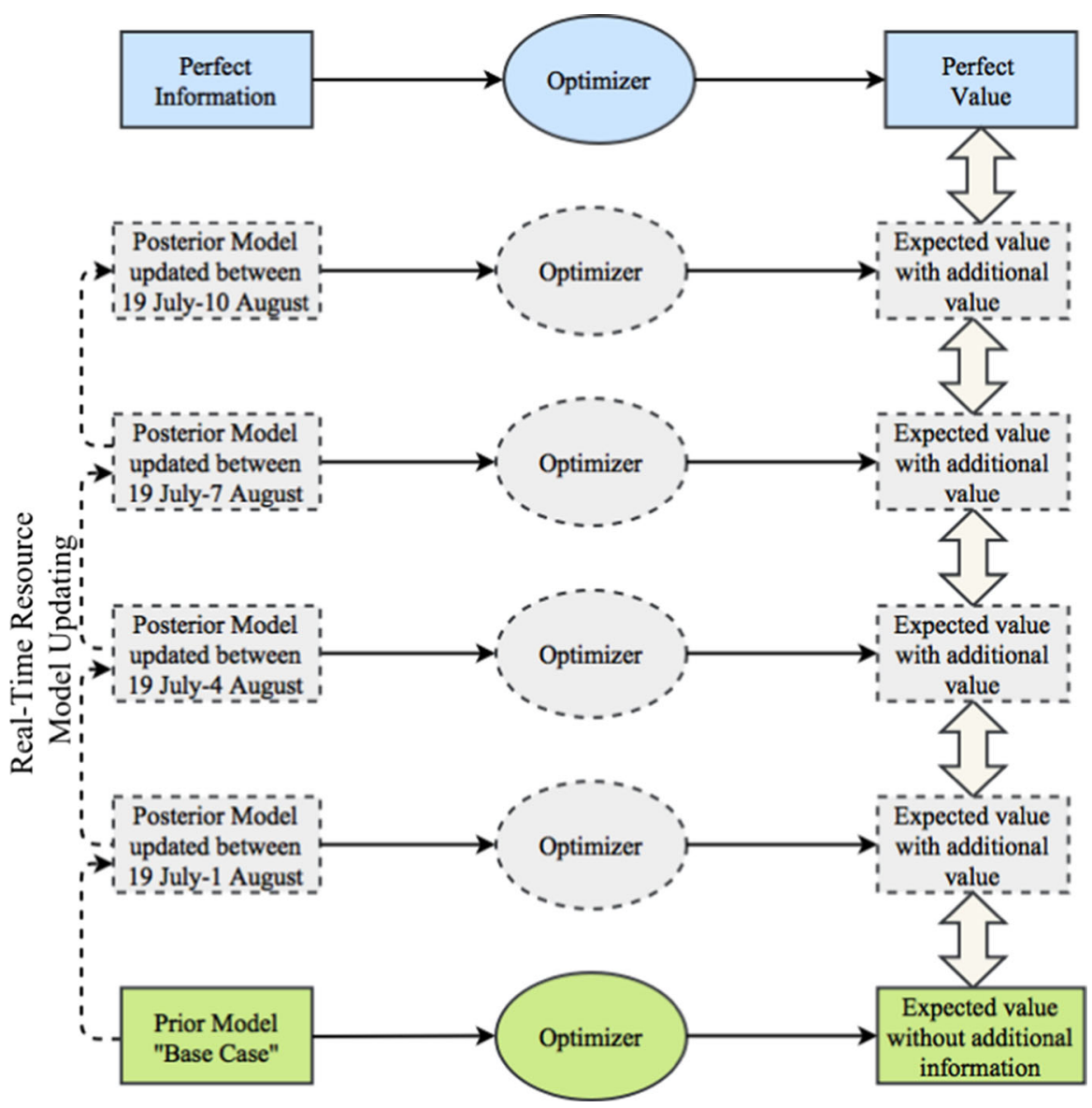

Fig. 9 VOI: experimental scheme for weekly job scheduling mine planning

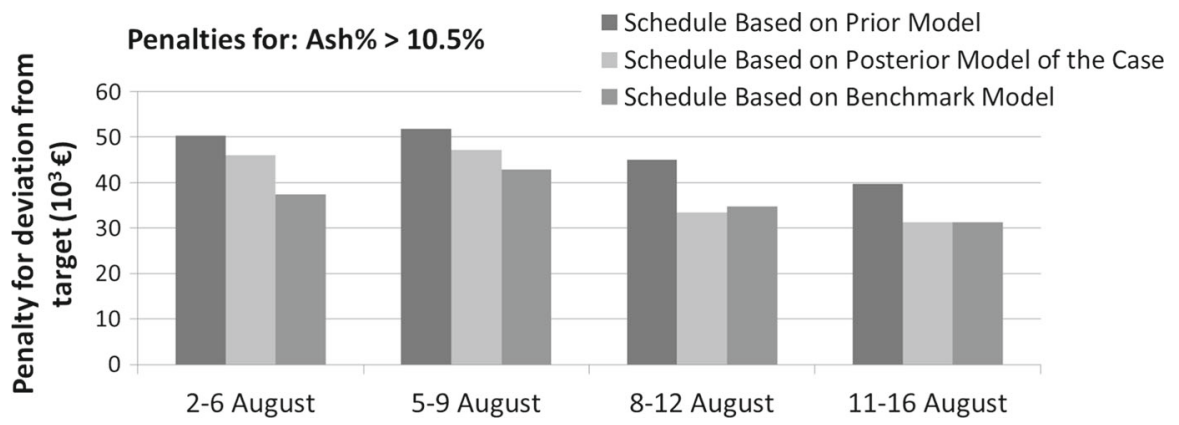

Fig. 10 Cost calculations of deviating from the target quality (ash \%)

VOI towards VOPI. Further, the following observations can be made: (i) the mean value of penalties for the schedule based on the prior model is $€ 47,000$, while periods vary between $€ 40,000$ and $€ 52,000$, (ii) penalties for the schedule based on the 


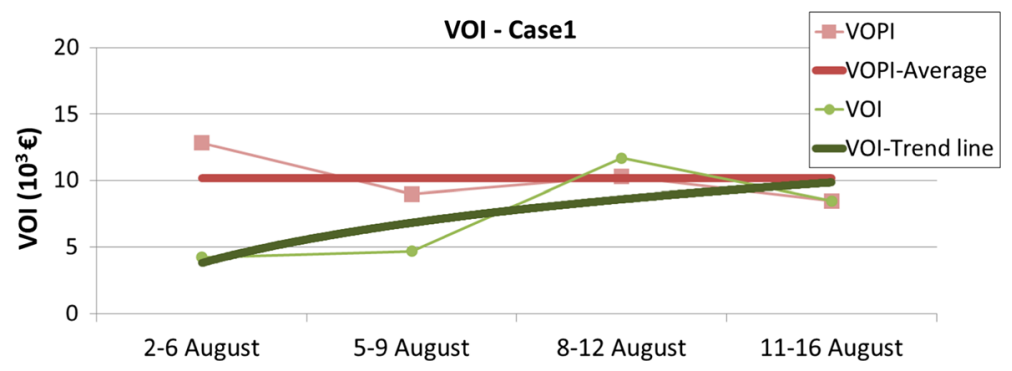

Fig. 11 VOI

posterior model gradually decrease from $€ 46,000$ to $€ 31,000$, (iii) the mean value of penalties for the schedule based on the benchmark model is $€ 37,000$, while periods vary between $€ 31,000$ and $€ 43,000$, (iv) thus, for this case study, the calculated VOPI is $€ 10,000$ and the calculated VOI moves from $€ 4000$ to $€ 8000$ (Fig. 11), and (v) the above-mentioned VOI numbers will lead to approximately a $€ 300,000$ to $€ 600,000$ annual cost reduction or savings. Note that these savings are solely related to the weekly task scheduling application applied to the upper limit of the ash content. In Fig. 11, the trend line of the VOI illustrates the benefit of using a combination of the resource model updating algorithm and the mine optimizer ("closed-loop" optimization). With each iteration of updating, the mine schedule optimization penalties decrease, thus VOI increases.

\subsection{Short-Term Mine Planning}

Tables 3 and 4 summarize the optimization model's parameters used in this case study. The model starts with a predefined state of the open-cut mine and plans the exploitation

Table 3 Defined quality bandwidths for the lignite product

\begin{tabular}{llll}
\hline & $Q_{\min }$ & $Q_{\text {target }}$ & $Q_{\text {max }}$ \\
\hline Ash $(\%)$ & 0 & 10.7 & 15 \\
\hline
\end{tabular}

Table 4 Information about the optimization model parameters for the deployed equipment

\begin{tabular}{llll}
\hline Excavator & $\begin{array}{l}\text { Operating hours } \\
(\mathrm{h} / \text { week })^{\mathrm{a}}\end{array}$ & Waste $\left(\mathrm{m}^{3} / \mathrm{h}\right)^{\mathrm{a}}$ & ${\text { Lignite }\left(\mathrm{m}^{3} / \mathrm{h}\right)^{\mathrm{a}}}^{\mathrm{a}}$ \\
\hline Excavator 1 & 4000 & 7050 & 4700 \\
Excavator 2 & 2800 & 3880 & 3170 \\
Excavator 3 & 2300 & 1645 & 2000 \\
Excavator 4 & 2500 & 2820 & 3170 \\
Excavator 5 & 4000 & 1410 & 1530 \\
\hline
\end{tabular}

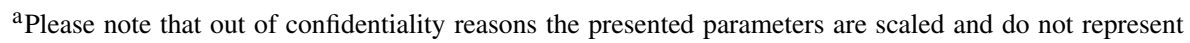
reality 


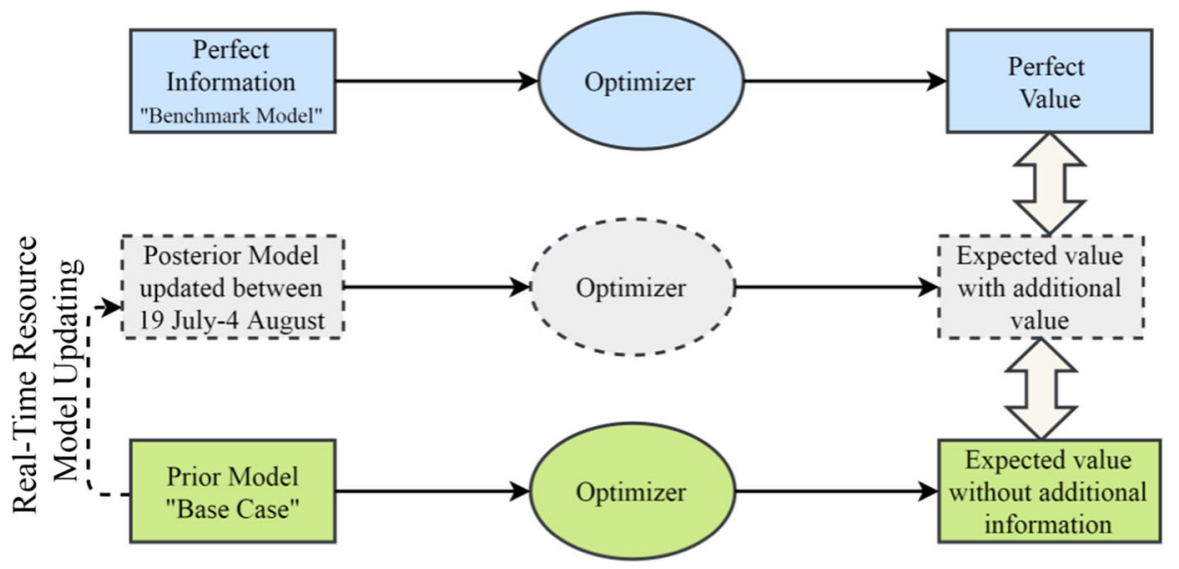

Fig. 12 VOI: experimental scheme for short-term mine planning

on five benches for four time periods (weeks). A total of 200,000 tons of lignite per time period (week) need to be supplied with a defined quality bandwidth for the ash content (Table 3).

To penalize exceeding or a shortfall with respect to defined lignite quality limits $Q_{\min }$ and $Q_{\max }$, a slope coefficient $\left(\mathrm{sl}_{1}, \mathrm{sl}_{4}\right)$ of " \pm 1.3 " is used (Fig. 3b). This represents 1.3 units of monetary value per unit deviation (€/ash $\% \times t)$. Within the quality limits, a slope coefficient $\left(\mathrm{sl}_{2}, \mathrm{sl}_{3}\right)$ of " \pm 1 " is used. For penalizing over- or underproduction, a slope coefficient of 40 or 80 , respectively, is applied per $10^{6}$ tons (Fig. 3a). As a result, this objective gets a higher priority, and the production scheduling of lignite of better quality but with an insufficient amount is avoided.

The optimizer is applied to a selection of the resource models defined in Sect. 5.2. In total, there are three different optimized mining plans for an optimization period of 4 weeks, 2-29 August: (i) optimized mining plan achieved by applying the optimizer to the prior resource model, (ii) optimized mining plan achieved by applying the optimizer to the posterior resource model (which is updated between 19 July and 4 August) and (iii) optimized mining plan achieved by applying the optimizer to the benchmark resource model (which is updated between 19 July and 13 August).

Next, these obtained best schedules are applied to the benchmark resource model (Fig. 12). This is done to see the improvements during the mining operations, when using the prior model, the most current model and the benchmark model. Similar to the weekly job scheduling mine planning experiments, the benchmark model is assumed to be the reality since it is the most up-to-date and, therefore, the most precise resource model. An approximation of VOPI is calculated between the benchmark and the prior model, whereas the VOI is calculated between the posterior model (4th August model; updated between 19 July and 4 August) and the prior model. Figure 13 shows the optimized mining sequences for the three different mining plans calculated by the optimizer. It is expected that one would observe more mining sequence differences between the mining plans created using the prior and the benchmark resource model than between the mining plans created using the benchmark and the posterior (updated 

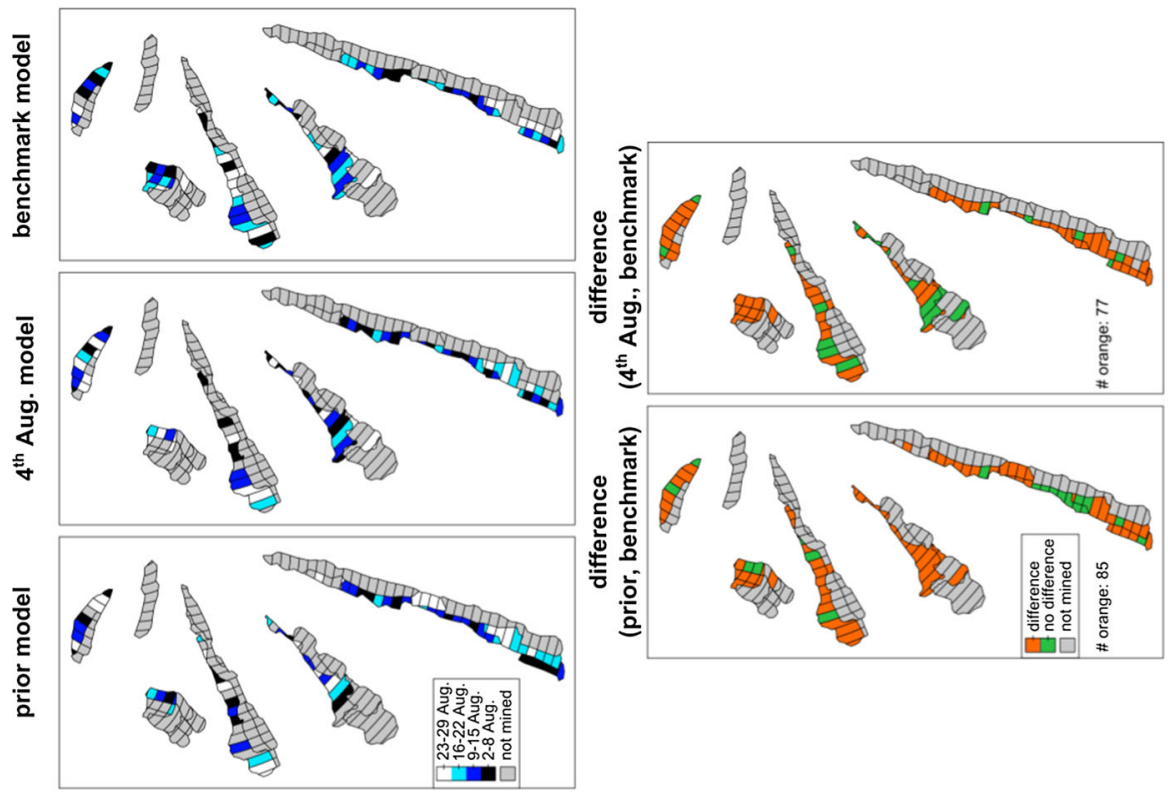

Fig. 13 Illustration of the optimized mining sequences and differences to the benchmark model
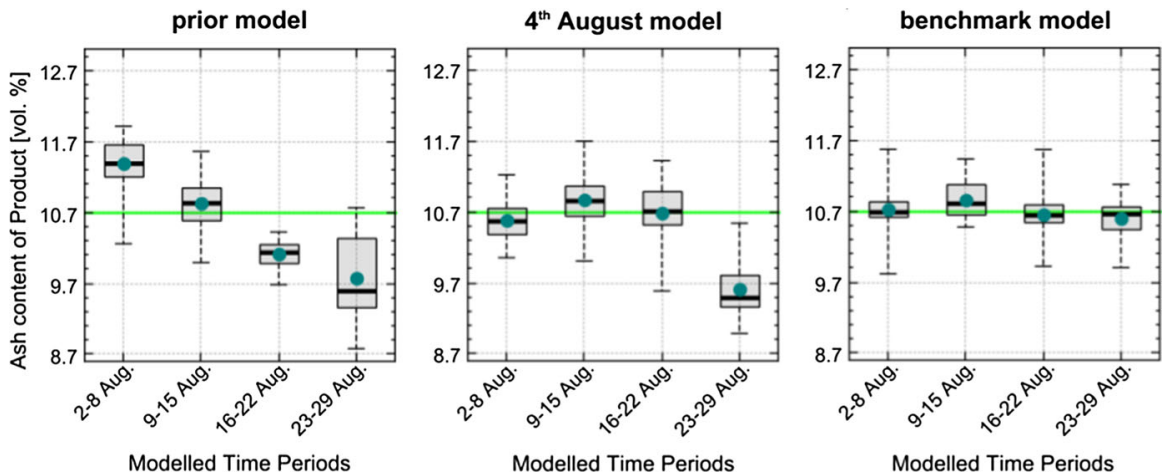

Fig. 14 Box plots for quality ash of the weekly scheduled lignite

till 4th August) resource model. A difference in the mining sequence means that either a block is scheduled for mining in different extraction periods in the two mining plans in question or that a block is scheduled for mining in one mining plan but not in the other. There are 85 differences in the optimized mining sequences determined using the prior and the benchmark resource model. For the optimized mining sequences, determined using the 4th August and the benchmark resource model, there are only 77 differences.

Figure 14 presents the lignite's ash content for each time period calculated by applying the optimized mining plans to the benchmark resource model. When the optimized mining plan is calculated based on a more accurate/up-to-date resource 
Penalties for: Ash\% $\neq 10.7 \%$

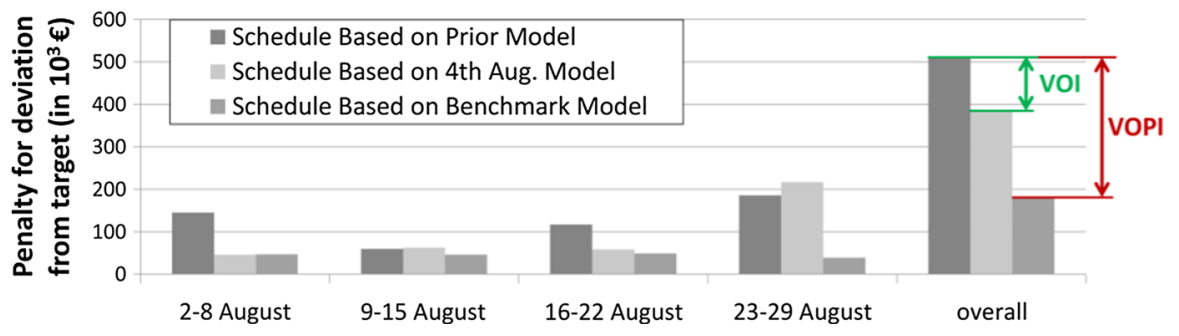

Fig. 15 Cost calculations of deviating from the target quality (ash \%)

model, the following observations can be made: (i) a better fitting of the average ash values to the target ash value of $10.7 \%$ is achieved, (ii) a better fitting of the average ash values to the target ash value area (defined from 0 to $15 \%$ ) is accomplished and (iii) a decrease in the uncertainty range is attained.

To quantify these findings and the VOI, penalties for deviating from the target ash value are used (Fig. 15). The darkest column represents the calculated penalties for the best mining plan, which is optimized based on the prior resource model, and later, this mining plan is applied to the benchmark resource model. The lightest column represents the calculated penalties for the best mining plan which is optimized based on the posterior resource model (4th August) of that case, and later, this mining plan is applied to the benchmark resource model. The medium darkest column represents the calculated penalties for the best mining plan which is optimized by using the benchmark resource model, and later, this mining plan is applied to the benchmark resource model. A decrease in penalties, when the optimized mining plan is calculated based on a more accurate/up-to-date resource model, is expected to be observed. When looking at each time step individually, this is not always the case. Since the optimizer calculates an overall best mining plan, single time steps can show a different behaviour for the penalty than the overall penalty of the entire optimization period (last entry in Fig. 15). For the overall penalty, the expected behaviour is observed: The VOI is $€ 124,110$, whereas the VOPI is $€ 327,090$. These significant benefits of the resource model updating framework are achieved for only one quality parameter, for a very short optimization time frame (in comparison to the life of mine) with limited localized updates. Therefore, annual cost reductions or savings between $€ 1.1$ and $€ 1.4$ Mio could be approximated. For other parameters, like the calorific value or sulphur content, which also have a major impact on the lignite quality and consequently the revenues, similar calculations can be made.

\section{Conclusions}

In this contribution, the added value of the real-time resource model updating concept is demonstrated by using a Value of Information (VOI) analysis. The expected economic benefits of additional information (due to the integration of the online sensor 
measurements into the resource model) is compared to different cases, where there is no additional information integrated into the process.

Using the resource model updating framework in combination with the mine optimizer, the performed case study proves that the deviations from the defined target quality are reduced. In summary, for weekly job scheduling, the calculated VOI varied between $€ 2000$ and $€ 8000$ for a five-day mining period. For short-term mine planning, the calculated VOI varied from $€ 92,000$ to $€ 124,000$ for 4 weeks of mining. These numbers will lead to approximately a $€ 100,000-€ 550,000$ annual cost reduction or saving using the weekly job scheduling optimization model. Using the short-term mine planning optimization model, which optimizes the extraction sequence, even higher annual cost reductions or savings could be possible $-€ 1.1$ Mio to $€ 1.4$ Mio.

This research demonstrates using the resource model updating framework leads to more accurate resource models at each iteration step. By having an up-to-date resource model, mine planning will yield better results and the efficiency of the mining process can be increased significantly. Deviations from the prior model can be processed and adapted quickly and efficiently. This would result in reaching the target product, which needs to be mined, more efficiently. Furthermore, if any deviations from the production targets are noticed and the plan needs to be changed, it can be done swiftly and will be based on a more accurate prediction of the mining environment.

Acknowledgements This research is a minor part of the Real-Time Reconciliation and Optimization in large open pit coal mines (RTRO-Coal) project and it is supported by the Research Fund for Coal and Steel of the European Union. RTRO-Coal, Grant agreement no. RFCR-CT-2013-00003.

Open Access This article is distributed under the terms of the Creative Commons Attribution 4.0 International License (http://creativecommons.org/licenses/by/4.0/), which permits unrestricted use, distribution, and reproduction in any medium, provided you give appropriate credit to the original author(s) and the source, provide a link to the Creative Commons license, and indicate if changes were made.

\section{References}

Aliyev E, Durlofsky LJ (2017) Multilevel field development optimization under uncertainty using a sequence of upscaled models. Math Geosci 49(3):307-339

Barnes RJ (1986) The cost of risk and the value of information in mine planning. In: Proceedings of 19th APCOM conference at the Pennsylvania State University. Society of Mining Engineers, Littleton, Colorado, pp 459-469

Barros EGD, Van den Hof PMJ, Jansen JD (2016) Value of information in closed-loop reservoir management. Comput Geosci 20(3):737-749

Benndorf J (2015) Making use of online production data: sequential updating of mineral resource models. Math Geosci 47(5):547-563

Benndorf J, Dimitrakopoulos R (2013) Stochastic long-term production scheduling of iron ore deposits: integrating joint multi-element geological uncertainty. J Min Sci 49(1):68-81

Benndorf J, Yueksel C, Soleymani MS, Rosenberg H, Thielemann T, Mittmann R, Lohsträter OM, Lindig M, Minnecker C, Donner R, Naworyta W (2015) RTRO-coal: real-time resource-reconciliation and optimization for exploitation of coal deposits. Minerals 5(3):546-569

Bhattacharjya D, Eidsvik J, Mukerji T (2010) The value of information in spatial decision making. Math Geosci 42(2):141-163

Bickel JE, Gibson RL, McVay DA, Pickering S, Waggoner JR (2008) Quantifying 3D land seismic reliability and value. SPE Reserv Eval Eng 11(5):832-841 
Bratvold RB, Bickel JE, Lohne HP (2009) Value of information in the oil and gas industry: past, present, and future. SPE Reserv Eval Eng 12(04):630-638

Dimitrakopoulos R (2011) Stochastic optimization for strategic mine planning: a decade of developments. J Min Sci 47(2):138-150

Dimitrakopoulos R, Ramazan S (2004) Uncertainty based production scheduling in open pit mining. SME Trans 312:106-112

Eidsvik J, Ellefmo SL (2013) The value of information in mineral exploration within a multi-gaussian framework. Math Geosci 45(7):777-798

Eidsvik J, Mukerji T, Bhattacharjya D (2016) Value of information in the earth sciences: integrating spatial modeling and decision analysis. Cambridge University Press, Cambridge

Goodfellow R, Dimitrakopoulos R (2017) Simultaneous stochastic optimization of mining complexes and mineral value chains. Math Geosci 49(3):341-360

Grayson CJ (1960) Decisions under uncertainty: drilling decisions by oil and gas operators. Published by Harvard Business School, Division of Research, Boston

Houck RT (2004) Predicting the economic impact of acquisition artifacts and noise. Lead Edge 23(10):1024-1031

Howard RA (1966) Information value theory. IEEE Trans Syst Sci Cybern 2(1):22-26

Liu X, Reynolds AC (2017) Robust gradient-based multiobjective optimization for the generation of well controls to maximize the net-present-value of production under geological uncertainty. Math Geosci 49(3):361-394

Matheson JE (1990) Using influence diagrams to value information and control. In: Oliver RM, Smith JQ (eds) Proceedings of influence diagrams belief nets and decision analysis. University of California, Oakland, pp 25-63

Mollema H (2015) Investigation into simulation based optimization of a continuous mining operation. Unpubl. MSc Thesis, Delft University of Technology, Delft

Newendorp PD (1975) Decision analysis for petroleum exploration. Petroleum Publishing Co., Tulsa

Peck J, Gray J (1999) Mining in the 21st century using information technology. CIM Bull 92(1032):56-59

Phillips J, Newman AM, Walls MR (2009) Utilizing a value of information framework to improve ore collection and classification procedures. Eng Econ 54(1):50-74

Raiffa H (1968) Decision analysis: introductory lectures on choices under uncertainty. McGraw-Hill, New York

Ramazan S, Dimitrakopoulos R (2007) Stochastic optimisation of long-term production scheduling for open pit mines with a new integer programming formulation. In: Orebody modelling and strategic mine planning, spectrum series, vol 14. The Australian Institute for Mining and Metalurgy, Melbourne, pp 359-365

Ramazan S, Dimitrakopoulos R (2013) Production scheduling with uncertain supply: a new solution to the open pit mining problem. Optim Eng 14(2):361-380

Steagall DE, Gomes JAT, De Oliveira RM, Ribeiro N, Queiroz RQ, Carvalho MJ, Souza CZ (2005) How to estimate the value of the information (VOI) of a 4D seismic survey in one offshore giant field. In: SPE annual technical conference and exhibition, 9-12 October, Dallas, Texas

Stibolt R, Lehman J (1993) The value of a seismic option. In: SPE hydrocarbon economics and evaluation symposium, society of petroleum engineers, 29-30 March, Dallas, Texas

Wambeke T, Benndorf J (2015) Data assimilation of sensor measurements to improve production forecasts in resource extraction. In: Proceedings of the 17th annual conference of the international association for mathematical geosciences, IAMG2015, Freiberg, Germany, pp 236-245

Wambeke T, Benndorf J (2016) A simulation-ased geostatistical approach to real-time reconciliation of the grade control model. Math Geosci 49(1):1-37

Yüksel C, Benndorf J, Lindig M, Lohsträter O (2016a) Updating the coal quality parameters in multiple production benches based on combined material measurement: a full case study. Int J Coal Sci Technol 4(2):159-171

Yüksel C, Thielemann T, Wambeke T, Benndorf J (2016b) Real-time resource model updating for improved coal quality control using online data. Int J Coal Geol 162:61-73 